\title{
Clinicopathological Factors Affecting Survival and Recurrence after Initial Hepatectomy in Non-B Non-C Hepatocellular Carcinoma Patients with Comparison to Hepatitis B or C Virus
}

\author{
Yoshihiro Okuda, ${ }^{1}$ Shugo Mizuno, ${ }^{1}$ Taizou Shiraishi, ${ }^{2}$ Yasuhiro Murata, ${ }^{1}$ \\ Akihiro Tanemura, ${ }^{1}$ Yoshinori Azumi, ${ }^{1}$ Naohisa Kuriyama, ${ }^{1}$ Masashi Kishiwada, ${ }^{1}$ \\ Masanobu Usui, ${ }^{1}$ Hiroyuki Sakurai, ${ }^{1}$ Masami Tabata, ${ }^{1}$ Tomomi Yamada, ${ }^{3}$ and Shuji Isaji ${ }^{1}$ \\ ${ }^{1}$ Department of Hepatobiliary-Pancreatic and Transplant Surgery, Mie University School of Medicine, 2-174 Edobashi, Tsu, Mie 514- \\ 0001, Japan \\ ${ }^{2}$ Department of Oncologic Pathology, Mie University Graduate School of Medicine, Tsu, Mie 514-0001, Japan \\ ${ }^{3}$ Department of Translational Medical Science, Mie University School of Medicine, 2-174 Edobashi, Tsu, Mie 514-0001, Japan
}

Correspondence should be addressed to Shugo Mizuno; mizunos@clin.medic.mie-u.ac.jp

Received 12 December 2013; Revised 8 February 2014; Accepted 9 February 2014; Published 13 March 2014

Academic Editor: Mohammad Ahmad al-Shatouri

Copyright (C) 2014 Yoshihiro Okuda et al. This is an open access article distributed under the Creative Commons Attribution License, which permits unrestricted use, distribution, and reproduction in any medium, provided the original work is properly cited.

\begin{abstract}
We evaluated clinicopathological factors affecting survival and recurrence after initial hepatectomy in non-B non-C (NBNC) hepatocellular carcinoma (HCC) patients with comparison to hepatitis B or C virus, paying attention to relationship between alcohol consumption and histopathological findings. The medical records on the 201HCC patients who underwent initial hepatectomy between January 2000 and April 2013 were retrospectively reviewed. NBNC patients had higher prevalence of hypertension (47.4\%), diabetes mellitus (35.5\%), alcohol consumption (>20 g/day) (61.8\%), and preserved liver function than hepatitis B or C patients. The 5-year survival rate of NBNC patients (74.1\%) was significantly better than hepatitis B (49.1\%) or C (65.0\%) patients (NBNC versus $\mathrm{B}, P=0.031)$. Among the NBNC patients, there was no relationship between alcohol consumption and clinicopathological findings including nonalcoholic fatty liver disease activity score (NAS). However, the 5-year OS and RFS rates in the alcoholunrelated NBNC patients tend to be better than in the alcohol-related. By multivariate analysis, independent factors for OS in NBNC patients were Child-Pugh B/C, intrahepatic metastasis (im), and extrahepatic recurrence. NBNC patients, who were highly associated with lifestyle-related disease and preserved liver function, had significantly better prognosis compared to hepatitis B/C patients; however, there was no association between alcohol consumption and histopathological findings.
\end{abstract}

\section{Introduction}

Primary liver cancer including hepatocellular carcinoma (HCC) is the fifth most frequently diagnosed cancer worldwide [1], and chronic viral hepatitis and liver cirrhosis following hepatitis B virus (HBV) or hepatitis C virus (HCV) infection had been reported to be responsible for most HCCs [2]. Although Japan has had one of the highest incidence rates of HCC associated with chronic HCV infection [3], a nationwide follow-up survey by the Liver Cancer Study Group of Japan found that the proportion of hepatitis virus-related
HCC had decreased over the previous decade, possibly due to the promotion of antiviral therapy, whereas the number of other HCC patients (mostly non-B non-C HCC: NBNCHCC) had more than doubled during the same period from $6.8 \%$ to $17.3 \%[4]$.

It remains controversial whether NBNC-HCC patients have comparable prognosis to HCC patients with HBV or HCV. In the previous studies, NBNC-HCC patients had a poorer prognosis than hepatitis virus-related HCC patients because NBNC-HCCs were often detected at an advanced stage incidentally without followup [5-7]. In contrast, a few 
studies reported that the postoperative outcome of NBNC patients were better than that of $\mathrm{HBV}$ or $\mathrm{HCV}$ patients, because hepatitis virus-related patients had poor liver function, more advanced tumors, and multicentric carcinogenesis in the remnant liver $[8,9]$. These conflicting results are considered to be due to the fact that the clinicopathological characteristics in NBNC patients still remain unclear because various clinical factors including age, gender, alcohol consumption, and DM are involved in the carcinogenesis and progression of NBNC-HCCs.

Nonalcoholic fatty liver disease (NAFLD) and nonalcoholic steatohepatitis (NASH) have recently assumed increasing attention for their relationship with HCC [1015]. Although laboratory test and radiographic findings may be suggestive of NAFLD, histological evaluation is still the gold standard for accurate diagnosis of NAFLD/NASH by assessing the degree of steatosis, the distinct necroinflammatory lesions and fibrosis of NASH, and distinguishing $\mathrm{NASH}$ from simple steatosis or steatosis with inflammation. Recently, feature-based semiquantitative scoring system of NAFLD, NAFLD activity score (NAS), was developed by the pathology committee of the NASH Clinical Research Network [16], and the feasibility of this score was reported as optimal scoring system for predicting steatohepatitis [17]. Nevertheless, there have been few reports examining the prevalence of steatohepatitis in NBNC-HCC patients and/or regarding the relationship between their surgical outcomes and degree of steatohepatitis.

The aim of the present study was to clarify the clinicopathological features of the NBNC-HCC patients who underwent initial hepatectomy by evaluating the factor affecting survival and recurrence after hepatectomy, paying attention to relationship between alcohol consumption and histopathological findings including NAS.

\section{Patients and Methods}

\subsection{Patient Groups}

2.1.1. Comparison among Three Groups according to Hepatitis Virus. We retrospectively reviewed a total of 201 primary HCC patients who consecutively underwent initial hepatectomy at the Mie University Hospital between January 2000 and April 2013. All patients were divided into the following three groups based on the presence of serum antigens/antibodies for hepatitis virus $\mathrm{B} / \mathrm{C}$ : group $\mathrm{B}(n=32)$ which were positive for HBs-Ag and negative for $\mathrm{HCV}-\mathrm{Ab}$; group $\mathrm{C}(n=93)$ which were negative for HBs-Ag and positive for $\mathrm{HCV}-\mathrm{Ab}$; and group $\mathrm{NBNC}(n=76)$ which were negative for both $\mathrm{HBs}-\mathrm{Ag}$ and $\mathrm{HCV}-\mathrm{Ab}$. In the present study, none of NBNC-HCC patients included the known etiologies such as primary biliary cirrhosis, other biliary cirrhosis (such as primary sclerosing cholangitis and secondary biliary cirrhosis), autoimmune hepatitis, metabolic diseases (Wilson's disease, hemochromatosis, and glycogen storage disease), congestive diseases including Budd-Chiari syndrome, and parasitic diseases.
2.1.2. Subgroup Analysis of NBNC-HCC according to Alcohol Consumption. According to the summary of talks presented at the American Association for the Study of Liver Diseases Clinical Single Topic Conference on NASH [18], a reasonable cut-off level of daily alcohol consumption, which would be the threshold at which steatohepatitis becomes alcohol-related, is $20 \mathrm{~g}$ ethanol. Therefore, all patients in group NBNC $(n=76)$ were further divided into the following two groups based on the daily alcohol consumption: NALP (nonalcoholic patients) $(n=30)$ in which alcohol consumption was less than $20 \mathrm{~g}$ ethanol/day and ALP (alcoholic patients) $(n=46)$ in which it was $20 \mathrm{~g}$ or more ethanol/day. A history of alcohol consumption was obtained from interviews with patients or their families.

2.2. Methods. We compared various factors in the three groups according to hepatitis virus in all patients and alcohol consumption in group NBNC in order to clarify clinicopathological characteristics of each group, including (1) lifestylerelated factors such as obesity, hypertension (HTN), and DM; (2) preoperative clinical factors such as neutrophil-tolymphocyte ratio (NLR), indocyanine green retention rate at 15 minutes (ICG R15), Child-Pugh class (A or B/C), tumor size (maximum diameter and more than $10 \mathrm{~cm}$ or less) on dynamic CT scan, Barcelona Clinic Liver Cancer (BCLC) stage (0/A or B/C) [19], and Milan criteria (within or beyond) [20]; (3) surgical factors such as surgical procedures (more than 2 sectionectomy or less) and surgical curability (R0 or R1/2); (4) pathological factors of the resected specimen such as intrahepatic metastasis (im) and histological findings of noncancerous liver; and (5) postoperative factors such as Clavien-Dindo classification for postoperative complications (I/II or III-V) [21] and posthepatectomy liver failure (PHLF) grade $(0 / \mathrm{A}$ or $\mathrm{B} / \mathrm{C})[22]$.

2.3. Definition of Obesity, Hypertension, DM, and Cigarette Smoking. Obesity was defined as body mass index (BMI) $\geqq 25 \mathrm{~kg} / \mathrm{m}^{2}$ according to the criteria of the Japan Society for the Study of Obesity [23]. Hypertension was diagnosed by a systolic blood pressure of more than $140 \mathrm{mmHg}$ and/or diastolic blood pressure of more than $90 \mathrm{mmHg}$ or by prescription of antihypertensive agents. DM was diagnosed according to the 2006 World Health Organization (WHO) criteria [24]. Cigarette smoking includes past history of smoking.

2.4. Definition of NLR. The NLR was calculated from the differential count by dividing the absolute neutrophil count by the absolute lymphocyte count. The cut-off level of NLR was defined as 4.0 according to the previous report [25].

2.5. Determination of the Type of Hepatectomy. After diagnosis of HCC, the most appropriate surgical procedure was selected based on the tumor characteristics and underlying the hepatic functional reserve of each patient by using ICGR15, LHL15, and hyaluronic acid according to our previous report [26]. 
2.6. Patient Follow-Up after Hepatectomy. Followup after surgery comprised periodic blood tests and monitoring of the tumor markers (serum $\alpha$-fetoprotein (AFP) level and desr-carboxyprothrombin (DCP) level). Dynamic CT images and/or MRI of the liver were carried out every 3-4 months until two years after hepatectomy and thereafter they were performed every 6 months. Chest CT, whole abdominal CT, brain MRI, and bone scintigraphy were done if recurrence of extrahepatic HCC was suspected.

2.7. Histological Examination of Noncancerous Liver in Group NBNC. In group NBNC, the noncancerous regions of the surgical specimens were stained with hematoxylin and eosin (H\&E) and Masson's trichrome and reevaluated by a single experienced pathologist (T.S.) who was unaware of the laboratory data and the clinical course, according to the National Institutes of Health-sponsored NASH Clinical Research Network system, which is called the Kleiner's scoring system [24] as follows. The elements of NAS and the stage of fibrosis were scored with separate scores for steatosis (0-3), hepatocellular ballooning (0-2), lobular inflammation (0-3), and fibrosis $(0-4)$. NAS was the sum of the first three features and ranged from 0 to 8 . Fibrosis according to the NAS was scored from 0 to 4 ( 0 : none, 1: perisinusoidal or periportal, 2 : perisinusoidal and periportal, and 3: bridging and 4: cirrhosis). NAS of 5 or more correlated with a diagnosis of NASH, and NAS of less than 3 were diagnosed as "not NASH." As the authors emphasized, the NAS was originally designed to assess overall histologic change before and after therapeutic intervention trials and was not intended as numeric values to replace a pathologist's diagnostic determination of steatohepatitis.

2.8. Statistical Analysis. All continuous values except for patient age are presented as medians and ranges (minimummaximum). Patient age is presented as mean \pm SD. Continuous variables were compared using Student's $t$-test or one-way analysis of variance (ANOVA) and Mann-Whitney $U$-test or Kruskal-Wallis method, and categorical variables were compared using Pearson's chi-squared test. The overall survival (OS) and recurrence-free survival (RFS) were calculated using the Kaplan-Meier method and tested using the log-rank test. In evaluating factors affecting OS, the Cox regression model with stepwise variable selection was used for multivariate analysis. Statistical data analysis was performed using the SPSS program, version 20.0 (SPSS, Chicago, Ill. USA). A $P$ value less than 0.05 was considered statistically significant.

\section{Result}

\subsection{Comparison among Three Groups according to Hepatitis Virus}

3.1.1. Preoperative Findings. The lifestyle-related and preoperative clinical findings in the three groups are listed in Table 1. The mean age of patients was the highest in group NBNC followed by groups $\mathrm{C}$ and $\mathrm{B}(P<0.001)$. The patients in group NBNC had significantly higher prevalence of hypertension
(47.4\%) and DM (35.5\%) than those in the other groups $(P=$ 0.004 and $P=0.041$, resp.). The incidence of patients with alcohol consumption of $20 \mathrm{~g} /$ day or more was the highest in group NBNC followed by groups $\mathrm{C}$ and $\mathrm{B}$. In the laboratory data, the platelet counts and PT were the highest in group NBNC followed by groups $\mathrm{C}$ and $\mathrm{B}(P=0.020$ and $P=0.002$, resp.). ICG R15 was the lowest in group NBNC followed by groups $\mathrm{B}$ and $\mathrm{C}(P=0.027)$. AFP levels were the lowest in group NBNC $(P<0.001)$ followed by groups $C$ and $B$. The percentage of the patients with tumors more than $10 \mathrm{~cm}$ in diameter was higher in groups NBNC (18.4\%) and B (21.9\%) than in group $\mathrm{C}(2.2 \%)(P=0.001$ and $P=0.001$, resp.). The proportion of the patients with tumors beyond Milan criteria was higher in groups NBNC (50.0\%) and B (46.9\%) than in group $\mathrm{C}(28.0 \%)(P=0.009)$.

3.1.2. Intraoperative, Pathological, and Postoperative Factors. As shown in Table 2, the proportion of the patients who needed more than two sectionectomy was significantly higher in groups $\mathrm{NBNC}$ and $\mathrm{B}$ than in group $\mathrm{C}(P=0.002)$. There were no significant differences in operative times, intraoperative blood loss, and surgical curability. In pathological examination, the proportion of simple nodular type was significantly lower in groups NBNC and B than in group C $(P=0.025)$, and the proportion of $\mathrm{vp}(+)$ was the second highest in group NBNC $(P=0.016)$. The proportion of NL in noncancerous liver was significantly higher in group NBNC than groups $\mathrm{B}$ and $\mathrm{C}$ and the proportion of $\mathrm{LC}$ was the lowest in group NBNC followed by groups $\mathrm{B}$ and $\mathrm{C}(P<0.001$ and $P=0.020$, resp.).

3.1.3. Cumulative Overall Survival and Recurrence-Free Survival. The survival curves of OS and RFS rates are shown in Figure 1. The median follow-up periods were 20 months (range, 0-136 months) for all patients. The 1- and 5-year OS rates were $88.3 \%$ and $74.1 \%$ in group NBNC, $88.9 \%$ and $65.0 \%$ in group $\mathrm{C}$ and $76.5 \%$, and $49.1 \%$ in group $\mathrm{B}$, respectively, showing significant difference between groups NBNC and $\mathrm{B}$ $(P=0.031)$. The $1-$ and 5-year RFS rates were $67.6 \%$ and $43.8 \%$ in group NBNC, $73.3 \%$ and $29.2 \%$ in group C, and $56.6 \%$ and $27.6 \%$ in group $B$, respectively. There was no significant difference in RFS rates between the three groups.

3.2. Subgroup Analysis of NBNC-HCC according to Alcohol Consumption. The lifestyle-related and preoperative clinical findings in the two subgroups are listed in Table 3. In NALP, the proportions of female and noncigarette smokers were significantly higher than those in ALP $(P=0.001$ and $P=$ 0.007 , resp.). As shown in Table 4, there were no significant differences in intraoperative, pathological, and postoperative factors between the two subgroups.

Table 5 shows the data based on the scores of steatosis, lobular inflammation, ballooning and fibrosis, the NAS, and NAS with fibrosis score. Moderate and severe steatosis, which is score of $2(33-66 \%)$ and $3(66 \%$ or more), was found only in 2 patients $(6.6 \%)$ in NALP and 2 patients $(4.3 \%)$ in ALP. Furthermore, NASH, which was defined as NAS of 5 or more, was found in 2 patients $(6.7 \%)$ in NALP. There were no 


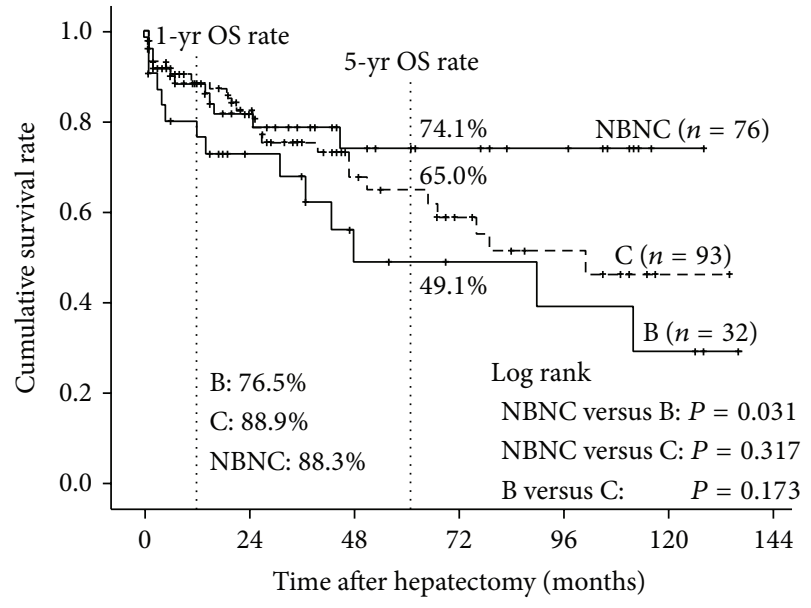

(a)

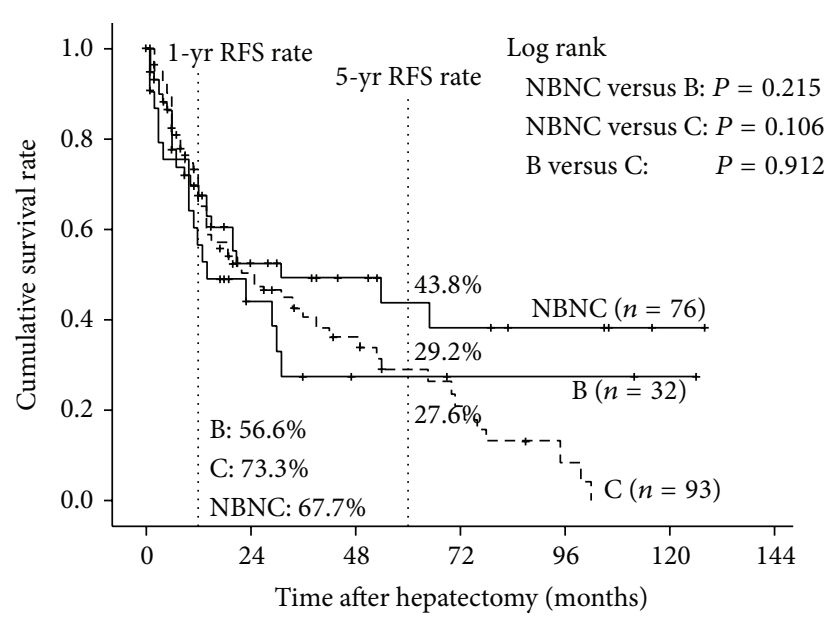

(b)

FIGURE 1: Comparisons of overall survival and recurrence-free survival rates after hepatectomy between patients in groups B, C, and NBNC. (a) Overall survival. The survival rate of NBNC patients (unbroken thick line) was significantly better than that of B patients (unbroken line, $P=0.031$ ). (b) Recurrence-free survival. There were no significant differences in survival rates of three groups.

TABLE 1: Lifestyle-related and preoperative clinical factors.

\begin{tabular}{|c|c|c|c|c|}
\hline & $\mathrm{B}(n=32)$ & $\mathrm{C}(n=93)$ & $\operatorname{NBNC}(n=76)$ & $P$ value \\
\hline Age (years) & $61.1 \pm 13.6$ & $67.2 \pm 6.6$ & $71.0 \pm 9.2$ & $<0.001$ \\
\hline Gender (male/female) & $23 / 9$ & $70 / 23$ & $67 / 9$ & 0.058 \\
\hline Obesity & $3(9.4 \%)$ & $17(18.3 \%)$ & $15(19.7 \%)$ & 0.412 \\
\hline Hypertension & $6(18.8 \%)$ & $26(28.0 \%)$ & $36(47.4 \%)$ & 0.004 \\
\hline $\mathrm{DM}$ & $5(12.0 \%)$ & $20(21.5 \%)$ & $27(35.5 \%)$ & 0.041 \\
\hline Cigarette smoking & $19(59.4 \%)$ & $55(59.1 \%)$ & $47(61.8 \%)$ & 0.933 \\
\hline Ethanol $\geqq 20 \mathrm{~g}$ & $5(15.6 \%)$ & $38(40.9 \%)$ & $46(60.5 \%)$ & $<0.001$ \\
\hline Albumin (g/dL) & $3.6(2.3-4.5)$ & $3.7(2.8-4.5)$ & $3.8(2.5-5.0)$ & 0.104 \\
\hline Total bilirubin (mg/dL) & $0.5(0.2-1.2)$ & $0.5(0.2-1.4)$ & $0.5(0.2-1.3)$ & 0.873 \\
\hline Platelet count $\left(\times 10^{4} / \mathrm{mm}^{3}\right)$ & $14.0(4.3-48.9)$ & $16.1(4.4-51.0)$ & $19.2(6.7-51.6)$ & 0.020 \\
\hline NLR & $2.21(0.79-4.90)$ & $1.92(0.52-14.16)$ & $2.16(0.67-12.80)$ & 0.271 \\
\hline PT (\%) & $82.9(64.3-105.0)$ & $90.0(63.4-122.7)$ & $95.4(54.8-131.4)$ & 0.002 \\
\hline ICG R15 (\%) & $12.7(4.4-19.7)$ & $14.0(2.8-42.3)$ & $10.9(1.6-36.7)$ & 0.027 \\
\hline Child-Pugh B/C & $2(6.3 \%)$ & $5(5.4 \%)$ & $6(7.9 \%)$ & 0.802 \\
\hline $\mathrm{AFP}(\mathrm{ng} / \mathrm{mL})$ & $110.0(1-605100)$ & $14.0(2-11675)$ & $6.0(1-48157)$ & $<0.001$ \\
\hline $\mathrm{DCP}(\mathrm{mAU} / \mathrm{mL})$ & $267.0(10-174400)$ & $148.0(10-23950)$ & $87.0(11-85330)$ & 0.653 \\
\hline Multiple tumors & $10(31.3 \%)$ & $25(26.9 \%)$ & $22(28.9 \%)$ & 0.885 \\
\hline Tumor size $(\mathrm{cm})$ & $5.2(1.0-20.0)$ & $3.0(0.7-11.0)$ & $4.5(1.0-17.0)$ & 0.001 \\
\hline$\geqq 10 \mathrm{~cm}$ & $7(21.9 \%)$ & $2(2.2 \%)$ & $14(18.4 \%)$ & 0.001 \\
\hline BCLC stage $\mathrm{B} / \mathrm{C}$ & $8(25.0 \%)$ & $16(17.2 \%)$ & $18(23.7 \%)$ & 0.484 \\
\hline Beyond Milan criteria & $15(46.9 \%)$ & $26(28.0 \%)$ & $38(50.0 \%)$ & 0.009 \\
\hline Preoperative TACE & $17(53.1 \%)$ & $60(64.5 \%)$ & $42(55.3 \%)$ & 0.356 \\
\hline
\end{tabular}

DM: diabetes mellitus; NLR: neutrophil-to-lymphocyte ratio; PT: prothrombin time; ICG R15: indocyanine green retention rate at 15 minutes; AFP: serum $\alpha$ fetoprotein; DCP: des-r-carboxyprothrombin; BCLC: Barcelona Clinic Liver Cancer; TACE: transcatheter arterial chemoembolization. 
TABLE 2: Intraoperative, pathological and postoperative factors.

\begin{tabular}{|c|c|c|c|c|}
\hline & $\mathrm{B}(n=32)$ & $\mathrm{C}(n=93)$ & $\operatorname{NBNC}(n=76)$ & $P$ value \\
\hline$\geqq 2$ sentionectomy & $13(40.6 \%)$ & $17(18.3 \%)$ & $32(42.1 \%)$ & 0.002 \\
\hline Operative Time (min.) & $352(177-658)$ & $333(135-750)$ & $367(173-983)$ & 0.142 \\
\hline Blood loss (g) & $1137(38-8488)$ & $1182(5-8307)$ & $1226(200-36000)$ & 0.590 \\
\hline Curability R0 & $27(84.4 \%)$ & $88(94.6 \%)$ & $70(92.1 \%)$ & 0.181 \\
\hline Simple nodular type & $12(37.5 \%)$ & $54(58.1 \%)$ & $30(39.5 \%)$ & 0.025 \\
\hline Poorly differentiated & $6(18.8 \%)$ & $14(15.1 \%)$ & $6(7.9 \%)$ & 0.218 \\
\hline $\mathrm{fc}(+)$ & $24(75.0 \%)$ & $63(67.7 \%)$ & $44(57.9 \%)$ & 0.182 \\
\hline $\mathrm{vp}(+)$ & $17(53.1 \%)$ & $24(25.8 \%)$ & $28(36.8 \%)$ & 0.016 \\
\hline $\operatorname{vv}(+)$ & $5(15.6 \%)$ & $4(4.3 \%)$ & $7(9.2 \%)$ & 0.109 \\
\hline $\operatorname{im}(+)$ & $6(18.8 \%)$ & $7(7.5 \%)$ & $7(9.2 \%)$ & 0.181 \\
\hline Non-cancerous liver NL & $1(3.1 \%)$ & $1(1.1 \%)$ & $23(30.3 \%)$ & $<0.001$ \\
\hline $\mathrm{CH}$ & $17(53.1 \%)$ & $44(47.3 \%)$ & $29(38.2 \%)$ & 0.288 \\
\hline LC & $14(43.8 \%)$ & $48(51.6 \%)$ & $23(30.3 \%)$ & 0.020 \\
\hline Clavien-Dindo $\geqq$ III & $5(15.6 \%)$ & $19(20.4 \%)$ & $12(15.8 \%)$ & 0.688 \\
\hline PHLF B/C & $7(21.9 \%)$ & $28(30.1 \%)$ & $18(23.7 \%)$ & 0.526 \\
\hline 90-day mortality & $4(12.5 \%)$ & $6(6.5 \%)$ & $6(7.9 \%)$ & 0.552 \\
\hline Intrahepatic recurrence & $13(40.6 \%)$ & $47(50.5 \%)$ & $25(32.9 \%)$ & 0.068 \\
\hline Extrahepatic recurrence & $7(21.9 \%)$ & $8(8.6 \%)$ & $7(9.2 \%)$ & 0.096 \\
\hline
\end{tabular}

fc: formation of capsule; vp: microscopic portal vein invasion; vv: microscopic hepatic vein invasion; im: intrahepatic metastasis; NL: normal liver; CH: chronic hepatitis; LC: liver cirrhosis; PHLF: posthepatectomy liver failure.

TABLE 3: Lifestyle-related and preoperative clinical factors in Group NBNC according to alcohol consumption.

\begin{tabular}{|c|c|c|c|}
\hline & $\operatorname{NALP}(n=30)$ & $\operatorname{ALP}(n=46)$ & $P$ value \\
\hline Age (years) & $73.3 \pm 6.3$ & $69.7 \pm 10.9$ & 0.491 \\
\hline Gender (Male/Female) & $22 / 8$ & $45 / 1$ & 0.001 \\
\hline Obesity & $7(23.3 \%)$ & $8(17.4 \%)$ & 0.525 \\
\hline Hypertension & $13(43.3 \%)$ & $23(50.0 \%)$ & 0.569 \\
\hline $\mathrm{DM}$ & $11(36.7 \%)$ & $16(34.8 \%)$ & 0.867 \\
\hline Cigarette smoking & $13(43.3 \%)$ & $34(73.9 \%)$ & 0.007 \\
\hline Albumin (g/dL) & $3.7(2.5-5.0)$ & $3.9(2.8-4.6)$ & 0.658 \\
\hline Total Bilirubin (mg/dL) & $0.5(0.2-1.3)$ & $0.5(0.2-0.9)$ & 0.834 \\
\hline Platelet count $\left(\times 10^{4} / \mathrm{mm}^{3}\right)$ & $20.8(8.0-40.4)$ & $17.8(6.7-51.6)$ & 0.100 \\
\hline NLR & $2.12(0.87-12.80)$ & $2.15(0.79-7.43)$ & 0.461 \\
\hline PT (\%) & $93.0(54.8-131.4)$ & $100.5(63.4-131.4)$ & 0.714 \\
\hline ICG R15 (\%) & $11.4(0.3-29.0)$ & $9.9(1.6-31.5)$ & 0.124 \\
\hline $\operatorname{AFP}(\mathrm{ng} / \mathrm{mL})$ & $7.0(1-48157)$ & $5.0(1-7153)$ & 0.398 \\
\hline $\mathrm{DCP}(\mathrm{mAU} / \mathrm{mL})$ & $94.0(14-85330)$ & $80.0(11-15387)$ & 0.741 \\
\hline Child-Pugh B/C & $3(10.0 \%)$ & $3(6.5 \%)$ & 0.583 \\
\hline Multiple tumors & $10(33.3 \%)$ & $12(26.1 \%)$ & 0.496 \\
\hline Tumor size $(\mathrm{cm})$ & $5.0(1.0-15.0)$ & $3.8(1.2-17.0)$ & 0.229 \\
\hline$\geqq 10 \mathrm{~cm}$ & $6(20.0 \%)$ & $8(17.4 \%)$ & 0.774 \\
\hline BCLC stage $\mathrm{B} / \mathrm{C}$ & $9(30.0 \%)$ & $9(19.6 \%)$ & 0.296 \\
\hline Beyond Milan criteria & $19(63.3 \%)$ & $19(41.3 \%)$ & 0.060 \\
\hline Preoperative TACE & $15(50.0 \%)$ & $27(58.7 \%)$ & 0.456 \\
\hline
\end{tabular}

NALP: non-alcoholic patients; ALP: alcoholic patients; DM: diabetes mellitus; NLR: neutrophil-to-lymphocyte ratio; PT: prothrombin time; ICG R15: indocyanine green retention rate at 15 minutes; AFP: serum $\alpha$-fetoprotein; DCP: des-r-carboxyprothrombin; BCLC: Barcelona Clinic Liver Cancer; TACE: transcatheter arterial chemoembolization. 
TABLE 4: Intraoperative, pathological and postoperative factors in Group NBNC according to alcohol consumption.

\begin{tabular}{lccc}
\hline & NALP $(n=30)$ & ALP $(n=46)$ & $P$ value \\
\hline$\geqq 2$ sentionectomy & $13(43.3 \%)$ & $19(41.3 \%)$ & 0.861 \\
Operative Time (min.) & $385(173-983)$ & $340(202-572)$ & 0.073 \\
Blood loss (g) & $1182(200-36000)$ & $1280(200-33478)$ & 0.663 \\
Curability R0 & $29(96.7 \%)$ & $41(89.1 \%)$ & 0.449 \\
Simple nodular type & $9(30.0 \%)$ & $21(45.7 \%)$ & 0.172 \\
Poorly differentiated & $2(6.7 \%)$ & $4(8.7 \%)$ & 0.748 \\
fc(+) & $18(60.0 \%)$ & $26(56.5 \%)$ & 0.764 \\
vp(+) & $12(40.0 \%)$ & $16(34.8 \%)$ & 0.645 \\
vv(+) & $2(6.7 \%)$ & $5(10.9 \%)$ & 0.536 \\
im(+) & $2(6.7 \%)$ & $5(10.9 \%)$ & 0.536 \\
Non-cancerous liver NL & $8(26.7 \%)$ & $15(32.6 \%)$ & 0.582 \\
CH & $12(40.0 \%)$ & $17(37.0 \%)$ & 0.789 \\
LC & $9(30.0 \%)$ & $5(10.9 \%)$ & 0.968 \\
Clavien-Dindo III & $7(23.3 \%)$ & $9(19.6 \%)$ & 0.145 \\
PHLF B/C & $9(30.0 \%)$ & $5(10.9 \%)$ & 0.296 \\
$90-$ day mortality & $1(3.0 \%)$ & $14(30.4 \%)$ & 0.234 \\
Intrahepatic recurrence & $11(36.7 \%)$ & $4(8.7 \%)$ & 0.572 \\
Extrahepatic recurrence & $3(10.0 \%)$ & 0.848 \\
\hline
\end{tabular}

NALP: non-alcoholic patients; ALP: alcoholic patients; fc: formation of capsule; vp: microscopic portal vein invasion; vv: microscopic hepatic vein invasion; im: intrahepatic metastasis; NL: normal liver; CH: chronic hepatitis; LC: liver cirrhosis; PHLF: posthepatectomy liver failure.

TABLE 5: Histopathological examination based on Kleiner's classification.

\begin{tabular}{|c|c|c|c|c|}
\hline Item & Score/Code & $\operatorname{NALP}(n=30)$ & $\operatorname{ALP}(n=46)$ & $P$ value \\
\hline \multirow{4}{*}{ Steatosis } & 0 & $24(80.0 \%)$ & $39(84.8 \%)$ & 0.588 \\
\hline & 1 & $4(13.3 \%)$ & $5(10.9 \%)$ & 0.745 \\
\hline & 2 & $1(3.3 \%)$ & $2(4.3 \%)$ & 0.824 \\
\hline & 3 & $1(3.3 \%)$ & $0(0.0 \%)$ & 0.213 \\
\hline \multirow{4}{*}{ Lobular inflammation } & 0 & $1(3.3 \%)$ & $0(0.0 \%)$ & 0.213 \\
\hline & 1 & $18(60.0 \%)$ & $34(73.9 \%)$ & 0.202 \\
\hline & 2 & $8(26.7 \%)$ & $11(23.9 \%)$ & 0.786 \\
\hline & 3 & $3(10.0 \%)$ & $1(2.2 \%)$ & 0.135 \\
\hline \multirow{3}{*}{ Ballooning } & 0 & $20(66.7 \%)$ & $35(77.1 \%)$ & 0.369 \\
\hline & 1 & $8(26.7 \%)$ & $7(15.2 \%)$ & 0.220 \\
\hline & 2 & $2(6.7 \%)$ & $3(6.5 \%)$ & 0.980 \\
\hline \multirow{5}{*}{ Fibrosis } & 0 & $2(6.7 \%)$ & $0(0.0 \%)$ & 0.076 \\
\hline & 1 & $10(33.3 \%)$ & $11(23.9 \%)$ & 0.369 \\
\hline & 2 & $3(10.0 \%)$ & $11(23.9 \%)$ & 0.126 \\
\hline & 3 & $6(20.0 \%)$ & $11(23.9 \%)$ & 0.689 \\
\hline & 4 & $9(30.0 \%)$ & $13(28.3 \%)$ & 0.870 \\
\hline NAS & & $2(0-5)$ & $2(1-4)$ & 0.290 \\
\hline NAS with fibrosis & & $4(0-9)$ & $4(2-8)$ & 0.996 \\
\hline
\end{tabular}

NALP: nonalcoholic patients; ALP: alcoholic patients; NAS: nonalcoholic fatty liver disease (NAFLD) activity score, NAS of 5 was found in two patients (6.7\%).

significant differences in all of the histopathological factors, NAS and NAS with fibrosis score between the two subgroups.

The survival curves of OS and RFS in NALP and ALP are shown in Figure 2. The median follow-up periods were 15 months (range, 0-128 months). The 1- and 5-year OS rates in NALP versus ALP were $89.4 \%$ versus $87.9 \%$ and $84.4 \%$ versus $68.7 \%$, respectively, showing no significant difference. The 1- and 5-year RFS rates in NALP versus ALP were $62.5 \%$ versus $71.9 \%$ and $57.3 \%$ versus $31.9 \%$, respectively, showing no significant difference. 


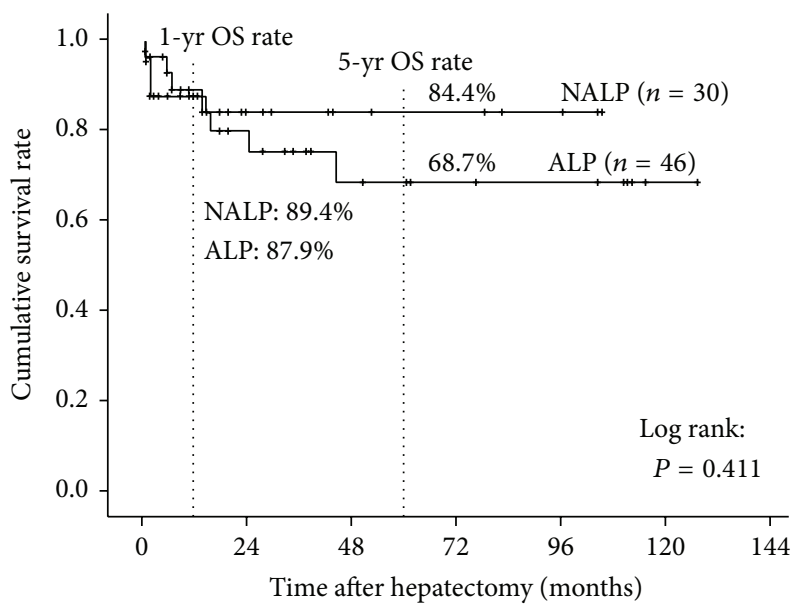

(a)

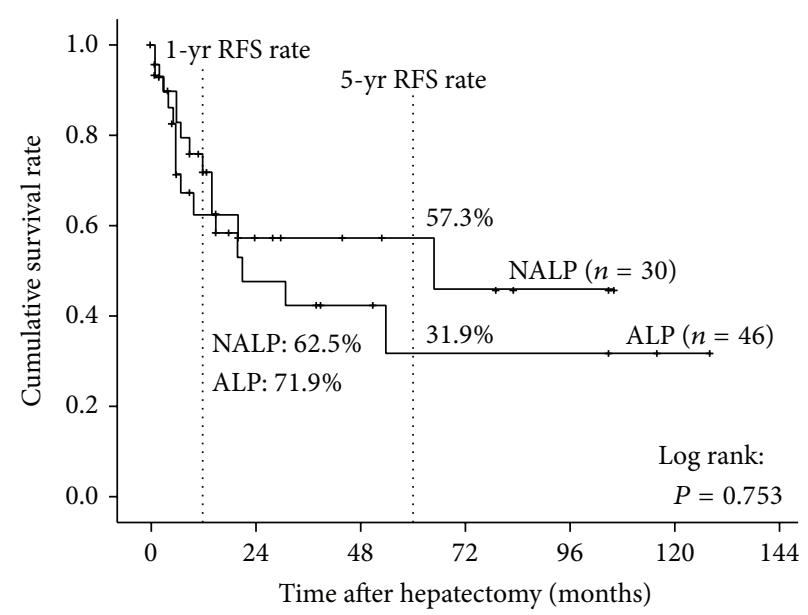

(b)

FIGURE 2: Comparisons of overall survival and recurrence-free survival rates after hepatectomy between patients in group NBNC. (a) Overall survival. There were no significant differences in survival rates of two subgroups. (b) Recurrence-free survival. There were no significant differences in survival rates of two subgroups.

TABLE 6: Multivariate analysis of factors contributing to overall survival and recurrence-free survival in group NBNC.

\begin{tabular}{lcc}
\hline Overall survival & Hazard ratio (95\% CI) & $P$ value \\
\hline Child-Pugh B/C & $19.667(1.346-287.340)$ & 0.029 \\
im $(+)$ & $31.064(1.965-491.024)$ & 0.015 \\
Extrahepatic recurrence & $31.717(1.261-797.764)$ & 0.036 \\
\hline
\end{tabular}

im: intrahepatic metastasis.

Table 6 shows the results obtained by multivariate analysis of factors influencing on OS. Child-Pugh B/C, im(+) and extrahepatic recurrence were identified as independent indicators of OS.

\section{Discussion}

To clarify the clinicopathological features of the NBNCHCC patients with initial hepatectomy, we first compared the patient- and tumor-related factors among the three groups $\mathrm{B}, \mathrm{C}$, and NBNC. In the patient-related factors, NBNC patients were characterized by association with lifestylerelated diseases (DM, HTN, and alcohol consumption) and preserved liver function (platelet counts, PT, ICG R15, and higher prevalence of NL in noncancerous liver).

To the best of our knowledge, there have been seven reports comparing clinicopathological features among the patients with hepatitis virus-related and nonhepatitis virusrelated HCCs all of which were from Japan after 2000, and, among them, four included less than 30 NBNC patients $[8,9,27,28]$ and the other three after 2012 included more than 60 NBNC patients $(n=60,129$, and 168, resp., [6, $29,30])$. Therefore, the results of our study were compared with those of the latter three reports. However, none of them focused on lifestyle-related diseases precisely, especially in NALFD/NASH. All of the previous three reports demonstrated that NBNC patients had much better preoperative liver function (platelet count and ICG R15), and Kudo et al. [30] reported that the incidence of liver cirrhosis was significantly lower in NBNC patient (34\%) than in HBV (52\%) and $\mathrm{HCV}(56 \%)$ patients, which were similar to our results.

As of the tumor-related factors, Kaibori et al. [6] reported that NBNC and HBV patients had significantly higher AFP and DCP levels than HCV patients, while the other two reports showed no difference in AFP and DCP levels among the three groups. The two reports demonstrated that tumor sizes were larger in NBNC (median $[\mathrm{cm}]: 5.57,5.8)$ and HBV (median [cm]: 5.43, 5.4) patients than in HCV (median [cm]: $3.55,4.0)$ patients, which were similar to our results. All of the three reports showed no significant differences in microvascular invasion and surgical margin among the three groups, which were similar to our results.

As of intraoperative and postoperative factors, the proportion of the patients with two sectionectomy was significantly higher in groups NBNC and B than in group C, reflecting tumor size in each group. There were no significant differences in operative times, intraoperative blood loss postoperative morbidity, and 90-day mortality among the three groups.

In the present study, the 5 -year OS rates were $74.1 \%$ in group NBNC, $65.0 \%$ in group C, and $49.1 \%$ in group B, showing significant difference between groups NBNC and $B$, while there was no significant difference in RFS rates between the three groups. The previous three reports $[6,29$, 30], however, demonstrated conflicting results: two reports showed significantly better survival in NBNC patients than in $\mathrm{HBV}$ and $\mathrm{HCV}$ patients, while the other report showed no survival difference in the three groups. In the former two reports $[6,30]$, since tumor-related factors did not differ significantly among the three groups, it was considered that good liver function at the initial hepatectomy might prevent early recurrence in NBNC patients. In the latter report [29], they suggested that the lack of difference in survival after curative resection might be because NBNC patients were associated with larger tumors but a better 
hepatic functional reserve. In our study, as of patient-related factors in NBNC patients, hepatic functional reserve was best preserved and the proportion of NL in noncancerous liver was the highest. While, as of tumor-related factors in NBNC patients, although AFP levels were the lowest, the percentages of the patients with tumors more than $10 \mathrm{~cm}$ in diameter and tumors beyond Milan criteria were high, showing similar percentages to HBV patients. Furthermore, the proportion of the patients who needed more than two sectionectomy was higher in groups NBNC and B than in group C. Taking these results together, it was considered that well-preserved liver function after initial hepatectomy in NBNC patients might contribute to survival benefit even if tumor recurrence occurred.

We furthermore performed subgroup analysis of NBNC patients based on alcohol consumption using cut-off level of ethanol $20 \mathrm{~g} /$ day. The proportions of female and noncigarette smokers were significantly higher in NALP than in ALP. Between the two subgroups, there were no significant differences in all of the other clinicopathological factors, including preoperative liver function and NAS. The OS and RFS rates in NALP were higher than those in ALP, although there were no statistical significant differences. This might be because the patient number was not so large and further investigation will be needed to clarify the influence of alcohol consumption on their prognosis. Kudo et al. [30] also analyzed the 163 NBNC patients by dividing the three groups according to alcohol consumption: nonalcoholic liver disease (non-ALD: ethanol less than $20 \mathrm{~g} /$ day), mild ALD (ethanol $20 \mathrm{~g}-80 \mathrm{~g} /$ day), and severe ALD (ethanol $80 \mathrm{~g} /$ day or more). They revealed that severe ALD was associated with being male, small tumor size, and LC, although they did not examine the degree of steatohepatitis. They concluded that preoperative excessive alcohol intake decreased DFS rate of HCC occurrence after surgery.

In multivariate analysis of factors influencing on OS in group NBNC, Child-Pugh $\mathrm{B} / \mathrm{C}$, im(+), and extrahepatic recurrence were identified as independent indicators of OS. Several previous studies on prognostic factors in NBNCHCC patients identified gender, serum albumin level, DCP, tumor size, tumor capsule, and tumor differentiation as significant independent factors for OS $[6,9,29-31]$. Therefore, in terms of OS, preoperative liver functions such as serum albumin and Child-Pugh class are important as patientrelated factor and degree of tumor malignancy as tumorrelated factor, showing the results similar to those in hepatitis virus-related HCC patients.

In conclusion, NBNC-HCC patients, who were characterized by association with lifestyle-related disease and preserved liver function, had significantly better prognosis compared to $\mathrm{HBV}$ and $\mathrm{HCV}$ patients. Significant prognostic factors in NBNC patients were preoperative poor hepatic functional reserve and tumor extension, which were similar to those in hepatitis virus-related HCC patients. In an attempt to clarify the association of NALFD/NASH with HCC by examining NAS in the resected specimens, however, we could not demonstrate any association.

\section{Conflict of Interests}

The authors declare that there is no conflict of interests regarding the publication of this paper.

\section{References}

[1] A. Jemal, F. Bray, M. M. Center, J. Ferlay, E. Ward, and D. Forman, "Global cancer statistics," CA Cancer Journal for Clinicians, vol. 61, no. 2, pp. 69-90, 2011.

[2] J. F. Perz, G. L. Armstrong, L. A. Farrington, Y. J. F. Hutin, and B. P. Bell, "The contributions of hepatitis B virus and hepatitis $\mathrm{C}$ virus infections to cirrhosis and primary liver cancer worldwide," Journal of Hepatology, vol. 45, no. 4, pp. 529-538, 2006.

[3] H. Tanaka, Y. Imai, N. Hiramatsu et al., "Declining incidence of hepatocellular carcinoma in Osaka, Japan, from 1990 to 2003," Annals of Internal Medicine, vol. 148, no. 11, pp. 820-826, 2008.

[4] T. Utsunomiya, M. Shimada, M. Kudo et al., "Nationwide study of 4741 patients with non-B non-C hepatocellular carcinoma with special reference to the therapeutic impact," Annals of Surgery, vol. 259, no. 2, pp. 336-345, 2014.

[5] Y. Takuma, K. Nouso, Y. Makino et al., "Outcomes after curative treatment for cryptogenic cirrhosis-associated hepatocellular carcinoma satisfying the Milan criteria," Journal of Gastroenterology and Hepatology, vol. 26, no. 9, pp. 1417-1424, 2011.

[6] M. Kaibori, M. Ishizaki, K. Matsui, and A. H. Kwon, "Clinicopathologic characteristics of patients with non-B non-C hepatitis virus hepatocellular carcinoma after hepatectomy," The American Journal of Surgery, vol. 204, no. 3, pp. 300-307, 2012.

[7] H. Akahoshi, N. Taura, T. Ichikawa et al., "Differences in prognostic factors according to viral status in patients with hepatocellular carcinoma," Oncology Reports, vol. 23, no. 5, pp. 1317-1323, 2010.

[8] A. Nanashima, T. Abo, Y. Sumida et al., "Clinicopathological characteristics of patients with hepatocellular carcinoma after hepatectomy: relationship with status of viral hepatitis," Journal of Surgical Oncology, vol. 96, no. 6, pp. 487-492, 2007.

[9] T. Wakai, Y. Shirai, N. Yokoyama, S. Nagakura, and K. Hatakeyama, "Hepatitis viral status affects the pattern of intrahepatic recurrence after resection for hepatocellular carcinoma," European Journal of Surgical Oncology, vol. 29, no. 3, pp. 266-271, 2003.

[10] M. M. Yeh and E. M. Brunt, "Pathology of nonalcoholic fatty liver disease," The American Journal of Clinical Pathology, vol. 128, no. 5, pp. 837-847, 2007.

[11] E. Hashimoto, S. Yatsuji, M. Tobari et al., "Hepatocellular carcinoma in patients with nonalcoholic steatohepatitis," Journal of Gastroenterology, vol. 44, no. 19, supplement, pp. 89-95, 2009.

[12] B. Q. Starley, C. J. Calcagno, and S. A. Harrison, "Nonalcoholic fatty liver disease and hepatocellular carcinoma: a weighty connection," Hepatology, vol. 51, no. 5, pp. 1820-1832, 2010.

[13] V. Ratziu, S. Bellentani, H. Cortez-Pinto, C. Day, and G. Marchesini, "A position statement on NAFLD/NASH based on the EASL 2009 special conference," Journal of Hepatology, vol. 53, no. 2, pp. 372-384, 2010.

[14] M. S. Ascha, I. A. Hanouneh, R. Lopez, T. A. Tamimi, A. F. Feldstein, and N. N. Zein, "The incidence and risk factors of hepatocellular carcinoma in patients with nonalcoholic steatohepatitis," Hepatology, vol. 51, no. 6, pp. 1972-1978, 2010. 
[15] K. Yasui, E. Hashimoto, Y. Komorizono et al., "Characteristics of patients with nonalcoholic steatohepatitis who develop hepatocellular carcinoma," Clinical Gastroenterology and Hepatology, vol. 9, no. 5, pp. 428-433, 2011.

[16] D. E. Kleiner, E. M. Brunt, M. van Natta et al., "Design and validation of a histological scoring system for nonalcoholic fatty liver disease," Hepatology, vol. 41, no. 6, pp. 1313-1321, 2005.

[17] M. Hjelkrem, C. Stauch, J. Shaw, and S. A. Harrison, "Validation of the non-alcoholic fatty liver disease activity score," Alimentary Pharmacology and Therapeutics, vol. 34, no. 2, pp. 214-218, 2011.

[18] B. A. Neuschwander-Tetri and S. H. Caldwell, "Nonalcoholic steatohepatitis: summary of an AASLD single topic conference," Hepatology, vol. 37, no. 5, pp. 1202-1219, 2003.

[19] J. M. Llovet, J. Fuster, J. Bruix, and Barcelona-Clínic Liver Cancer Group, "The Barcelona approach: diagnosis, staging, and treatment of hepatocellular carcinoma," Liver Transplantation, vol. 10, no. 2, supplement 1, pp. S115-S120, 2004.

[20] V. Mazzaferro, E. Regalia, R. Doci et al., "Liver transplantation for the treatment of small hepatocellular carcinomas in patients with cirrhosis," The New England Journal of Medicine, vol. 334, no. 11, pp. 693-699, 1996.

[21] D. Dindo, N. Demartines, and P. Clavien, "Classification of surgical complications: a new proposal with evaluation in a cohort of 6336 patients and results of a survey," Annals of Surgery, vol. 240, no. 2, pp. 205-213, 2004.

[22] N. N. Rahbari, O. J. Garden, R. Padbury et al., "Posthepatectomy liver failure: a definition and grading by the international study group of liver surgery (ISGLS)," Surgery, vol. 149, no. 5, pp. 713724, 2011.

[23] Examination Committee of Criteria for "Obesity Disease" in Japan and Japan Society for the Study of Obesity, "New criteria for "obesity disease" in Japan," Circulation Journal, vol. 66, no. 11, pp. 987-992, 2002.

[24] World Health Organization, Definition and Diagnosis of Diabetes Mellitus and Intermediate Hyperglycemia: Report of a WHO/IDF Consultation, World Health Organization, Lyon, France, 2006.

[25] T. Yoshizumi, T. Ikegami, S. Yoshiya et al., "Impact of tumor size, number of tumors and neutrophil-to-lymphocyte ratio in liver transplantation for recurrent hepatocellular carcinoma," Hepatology Research, vol. 43, no. 7, pp. 709-716, 2013.

[26] Y. Ohkura, S. Mizuno, M. Kishiwada et al., "Benefit of technetium-99m galactosyl human serum albumin scintigraphy instead of indocyanine green test in patients scheduled for hepatectomy," Hepatology Research, 2013.

[27] K. Noguchi, O. Nakashima, Y. Nakashima, K. Shiota, H. Nawata, and M. Kojiro, "Clinicopathologic study on hepatocellular carcinoma negative for hepatitis B surface antigen and antibody to hepatitis C virus," International Journal of Molecular Medicine, vol. 6, no. 6, pp. 661-665, 2000.

[28] Y. Yokoi, S. Suzuki, S. Baba, K. Inaba, H. Konno, and S. Nakamura, "Clinicopathological features of hepatocellular carcinomas (HCCs) arising in patients without chronic viral infection or alcohol abuse: a retrospective study of patients undergoing hepatic resection," Journal of Gastroenterology, vol. 40, no. 3, pp. 274-282, 2005.

[29] H. Nishikawa, A. Arimoto, T. Wakasa, R. Kita, T. Kimura, and Y. Osaki, "Comparison of clinical characteristics and survival after surgery in patients with non-B and non-C hepatocellular carcinoma and hepatitis virus-related hepatocellular carcinoma," Journal of Cancer, vol. 4, no. 6, pp. 502-513, 2013.
[30] A. Kudo, S. Tanaka, D. Ban et al., "Alcohol consumption and recurrence of non-B or non- $\mathrm{C}$ hepatocellular carcinoma after hepatectomy: a propensity score analysis," Journal of Gastroenterology, 2013.

[31] S. K. Kim, H. Marusawa, Y. Eso et al., "Clinical characteristics of non-B non-C hepatocellular carcinoma: a single-center retrospective study," Digestion, vol. 84, supplement 1, pp. 43-49, 2011. 


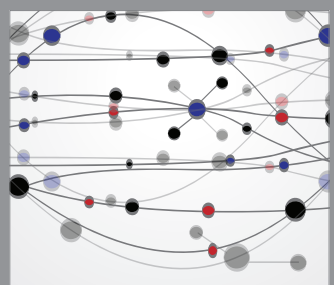

The Scientific World Journal
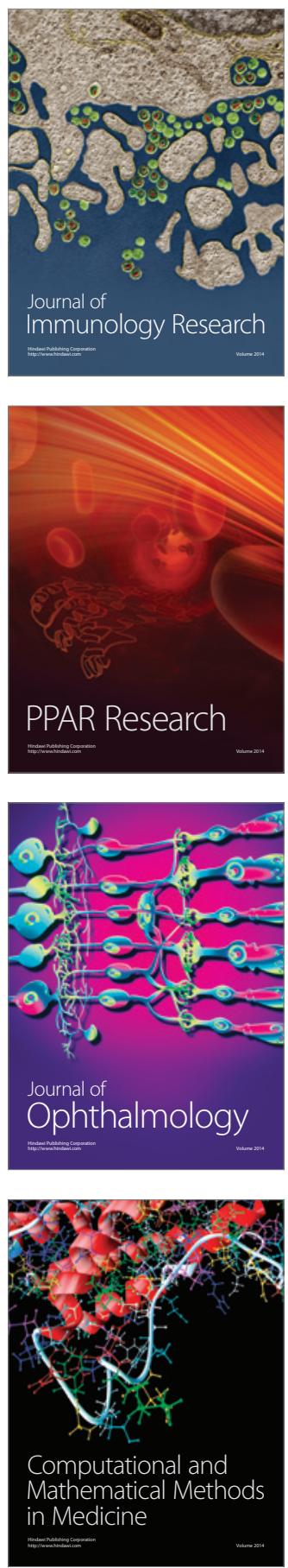

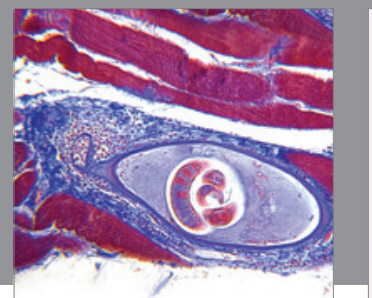

Gastroenterology

Research and Practice
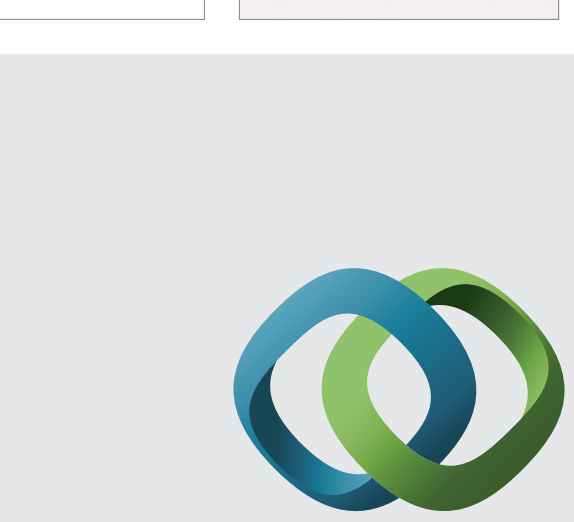

\section{Hindawi}

Submit your manuscripts at

http://www.hindawi.com
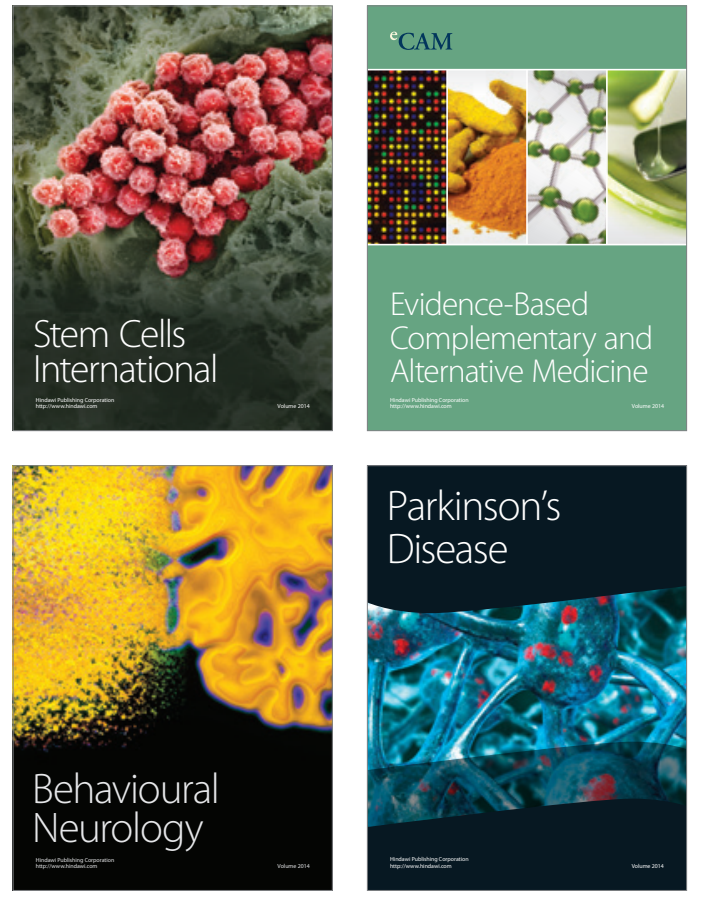
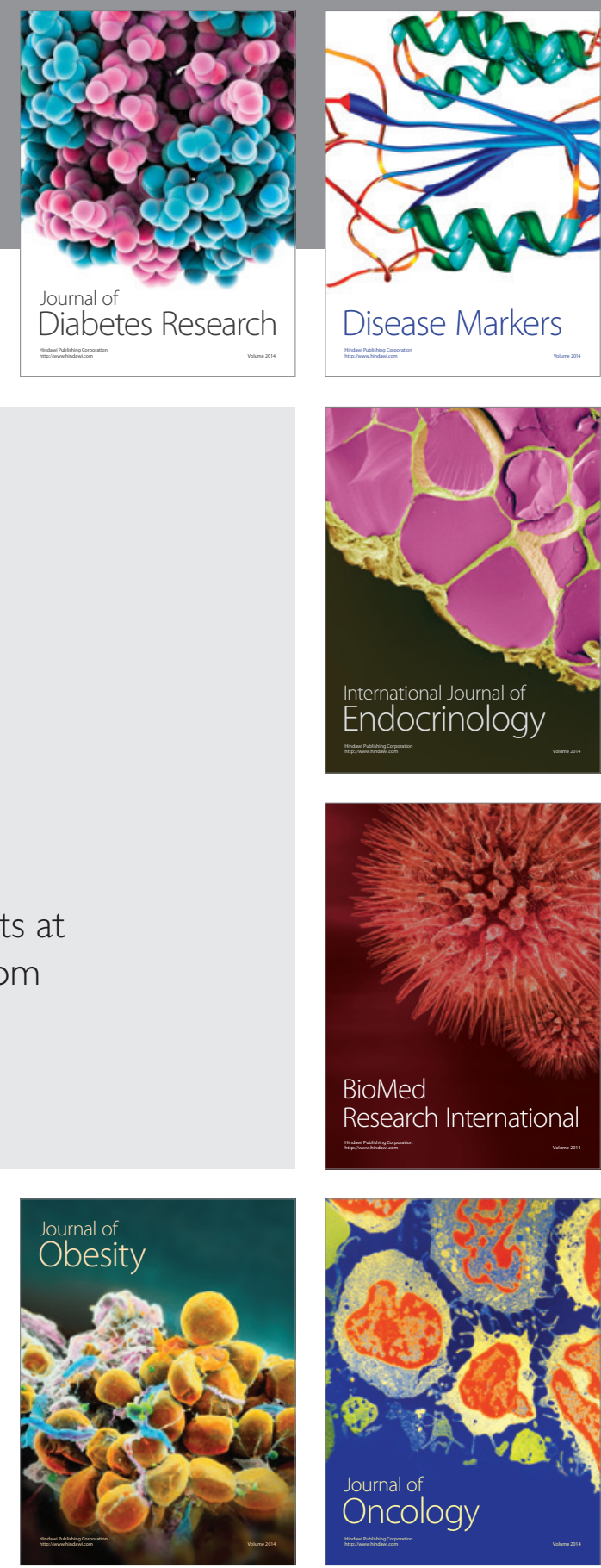

Disease Markers
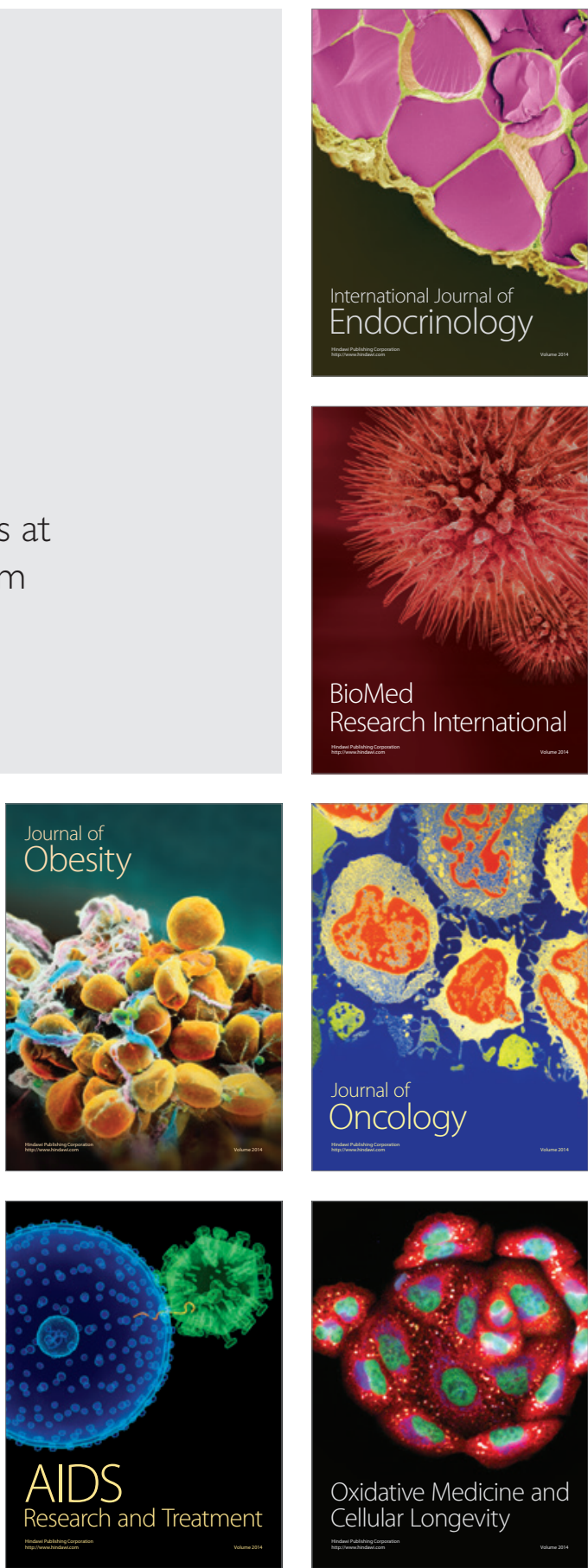